\title{
Food stall vendors' attitudes to whole grains in Singapore
}

\author{
Q. Chong and I.A. Brownlee \\ Newcastle University (Singapore), Human Nutrition Research Centre, 172A Ang Mo Kio Ave 8, Singapore
}

Like most other developed countries, the current intake of wholegrain foods in Singapore is low and infrequent ${ }^{(1)}$. Out-of-home food consumption is common in Singapore, particularly through traditional food markets or "hawker centres" that offer a wide range of inexpensive food options. One focus for increasing wholegrain food intake from the public health perspective is to increase the availability of out-of-home wholegrain options, particularly through hawker centre outlets ${ }^{(2)}$. However, this approach is only likely to be successful if the vendors (hawkers) actively engage in such programmes. The current study therefore aimed to evaluate the attitudes of hawkers to wholegrain foods.

Newcastle University Faculty of Science, Agriculture and Engineering Ethics Committee approved the current study. A total of 120 participants were recruited via purposive sampling to avoid an unequal geographical distribution of vendors across the island. Participants were approached outside of peak hours to ensure that they could effectively engage in the researcher-led, structured, questionnaire-based interview. Participants were questioned on their knowledge of whole grains, whether they sold, or have previously sold, wholegrain foods and their thoughts towards potentially selling whole grains at their stalls.

While most vendors $(n=97)$ were aware of what whole grains are, only c. $20 \%(n=26)$ provided whole grains at their stall. Stallholders that did not provide wholegrain options noted external issues with provision of wholegrain foods, such as customer demand and lack of control of menu provision (due to their stall being a franchise). Further internal barriers to provision of whole grains were also noted, such as concern about their reputation, lack of manpower of cooking skills to provide whole grains, or the increased potential for food wastage/spoilage. A number of shared external and internal barriers to wholegrain food provision were also noted to be shared between whole grain-providing vendors and those that did not provide these items. The factors included the suitability of wholegrain dishes to the cuisine their stall provided, issues with taste and texture acceptability by customers, higher running costs (as a result of ingredients cost, manpower and cooking needs). Of the 94 stall holders not providing whole grains currently, 35 had noted that they would be willing to try and sell wholegrain foods in the future. Factors that might motivate them to do so included having a better understanding of whole grains and increased consumer demand. The 26 vendors that already sold whole grains also agreed that higher demand from consumers would motivate them to offer more wholegrain options, as would higher amounts of manpower and finding more items that were suitable to the cuisine they were purveying.

In conclusion, this study has highlighted some major barriers to wholegrain food provision by hawkers in Singapore, as well as offering insight into how some of these barriers may be overcome.

1. Health Promotion Board (2010) National Nutrition Survey 2010.

2. Heath Promotion Board (2011) "The Healthier Hawker Programme" https://www.healthhub.sg/live-healthy/24/healthyhawkerfood. (accessed 27 March 2017) 